\title{
EL TRUEQUE SOLIDARIO: UNA ESTRATEGIA DE SUPERVIVIENCIA ANTE LA CRISIS ARGENTINA DE 2001
}

\author{
Manuela Fernández Mayo \\ mafermayo@ono.com \\ UNIVERSIDAD DE CÁDIZ, ESPAÑA
}

\begin{abstract}
RESUMEN
El artículo que presentamos analiza a través de los testimonios orales el fenómeno del trueque solidario, un mecanismo de contención social de aplicación masiva que junto con los planes sociales, ambos comparten su carácter de innovación frente a la crisis y su amplio alcance.

El trueque se presenta como una actividad, de gran convocatoria, surgida desde la sociedad civil, y aunque actualmente está en decadencia, cuando la crisis vivió sus días más agudos —en el año 2002 — llegó a reunir más de siete millones de personas a lo largo de la geografía argentina.
\end{abstract}

Palabras clave: Trueque solidario, contención social, sociedad civil. 


\begin{abstract}
The article we present analyzes through oral testimonies the phenomenon of solidary bartering, a social contention mechanism of massive application. Together with the social plans, the two share their character of innovation in the face of the crisis and its broad reach. Bartering is presented as an exceptionally widespread activity emerged from civil society. Although now in decline, during the period of sharpest crisis - in the year 2002 - it united more than seven million people across the full length of Argentinean territory.
\end{abstract}

Key words: Solidary bartering, social contention, civil society. 
Mientras en la economía mundial se registra el avance de las denominadas «monedas globales»—como el dólar, el euro, el yen— que tienden a «concentrar» todas las operaciones comerciales, en la Argentina de la crisis de 2001 se asistió a un proceso inverso, dado por un fuerte retroceso de la moneda de curso legal y un avance exponencial del trueque y de los medios de pago alternativos.

La caída de la producción, la baja del consumo y la recesión récord, que azotaban desde 1998 la economía argentina, alentaron la aparición de diversos mecanismos de sustitución del dinero real. Ese fenómeno alcanzó tanto a los gobiernos como a los agentes privados.

Hacia finales del año 2001 y comienzos de 2002, Argentina afrontaba una de las crisis más profundas de toda su historia. ${ }^{1}$ Si bien resulta complejo establecer un punto de partida de dicho desequilibrio, se puede ubicar su mayor visibilidad en los últimos meses del gobierno de Fernando de la Rúa, en un contexto social producto de más de diez años de políticas de ajuste estructural. $^{2}$

En este escenario adquirieron protagonismo diversas iniciativas de la sociedad civil, algunas totalmente nuevas, tales como asambleas barriales $\mathrm{y}$ cacerolazos; $\mathrm{y}$ otras preexistentes, con mayor protagonismo durante este periodo, como piquetes, microemprendimientos y proyectos vinculados con la economía social: experiencias de fábricas recuperadas, cooperativas de cartoneros, trueque, etc., además de la solidaridad, que llama la atención por su intensidad y por la novedad de muchas de sus fórmulas.

Sin desconocer esta multiplicidad de mecanismos organizativos surgidos desde la sociedad civil ni los diversos instrumentos económicos, sociales y políticos que el Estado ha utilizado para hacer frente a la crisis, en esta comunicación nos proponemos focalizar la atención sobre una de las dos estrategias que han tenido un alto nivel de masividad, 
especialmente durante el año 2002, y que estuvieron directamente asociadas con la necesidad de generar «contención social».

Una surgió como respuesta del Estado nacional: el Plan Jefas y Jefes de Hogar Desocupados; la otra, desde la sociedad civil: el trueque, que si bien su origen data de 1995 obtuvo relevancia en la agenda pública, estatal y mediática durante el año 2002.

Desarrolladas inicialmente por la clase media empobrecida, posteriormente se fueron integrando amplios sectores de las clases populares, nacieron como respuesta a la crisis económica, a la falta de trabajo y recursos; se fueron potenciando por la casi ausencia de dinero circulante debida al «corralito» ${ }^{3}$ bancario; y se convirtieron en el ámbito en que millones de ciudadanos buscaron obtener una digna economía de subsistencia. Hasta su declive a finales de 2002 se podía conseguir casi todo en los clubes de trueque; actuaron como estrategia de supervivencia necesaria para mucha gente que no tenía dinero.

Experimentos como el trueque con moneda propia ya se dieron con anterioridad en otros lugares del mundo y también en tiempos de crisis, consiguiendo respetables éxitos; ${ }^{4}$ pero lo que confiere originalidad al fenómeno del trueque argentino es su dimensión, ya que constituyó el fenómeno más grande a escala mundial.

Sus creadores sostienen que se trata de un estilo de vida y de una forma de reinserción ciudadana. Pero aunque resolvió los problemas inmediatos de mucha gente, el crecimiento exponencial de los clubes planteó serias dificultades y problemas que terminaron conduciéndolo a su decadencia. 


\section{BREVE HISTORIA DEL TRUEQUE ARGENTINO}

El origen de la red de trueque se remonta al $1 .^{\circ}$ de mayo de 1995 , en Bernal, localidad correspondiente al partido de Quilmes, en el conurbano bonaerense, cuando Horacio Covas, Carlos De Sanzo y Rubén Rávera, un grupo de vecinos ecologistas que ya habían puesto en marcha algunos emprendimientos productivos de huertas orgánicas, deciden reunir a vecinos del lugar para hacer frente a necesidades concretas surgidas de la falta de trabajo; el club reunió en sus comienzos a veinte personas, al estilo de un grupo de autoayuda inspirado en Alcohólicos Anónimos, para «... alcanzar un sentido de vida superior mediante el trabajo, la comprensión y el intercambio justo» y «responder a normas éticas y ecológicas antes que a los dictados del mercado, el consumismo y la búsqueda de beneficio a corto plazo» (Stancanelli 2002: 8).

Para celebrar sus reuniones eligieron las instalaciones abandonadas de la ex fábrica textil La Bernalesa. De acuerdo con el relato de los fundadores, la iniciativa estaba inspirada en la experiencia brasileña de la «Campaña contra el Hambre», liderada por Betinho, que había reunido de modo descentralizado a un conjunto de instituciones sociales y varias ONG.

La red de trueque surgió como resultado del encuentro de dos vertientes: una ecologista, el Programa de Autosuficiencia Regional, creada por De Sanzo y Ravera; y otra empresaria, la Red Profesional, de Horacio Covas. Esta asociación ayudó a potenciar ambas iniciativas, trasformándolas en una nueva propuesta, superadora y de mayor alcance. Más tarde, entre 1996-97, se concreta la incorporación de una tercera corriente, la Red de Intercambio de Saberes y Cibernética Social promovida por Heloísa Primavera y Carlos Del Valle, que hacen importantes aportes metodológicos, introduciendo técnicas de dinámica grupal explícita, incorporando los intercambios de saberes como una nueva modalidad, y 
poniendo énfasis en la capacitación permanente como condición para el crecimiento de la red (Leoni y Luzzi 2003: 15).

En Argentina, si bien el hambre no constituía un tema social relevante, sí comenzaba a serlo el desempleo, por lo que el trueque aparecía como una alternativa frente a una necesidad social. Se comenzaron a reunir los sábados, concurriendo aproximadamente unos sesenta participantes. Durante sus primeros seis meses de vida, el club de trueque de Bernal evolucionó en medio de una atmósfera de entusiasmo local: «... Por turno, los socios ingresábamos a un sector donde dejábamos diversos productos como tartas, empanadas, pizzas, artículos de vestimenta y artesanía, descontándose el consumo personal del saldo de la tarjeta [los datos se manejaban en planilla de cálculo]» (De Sanzo 1998: 9).
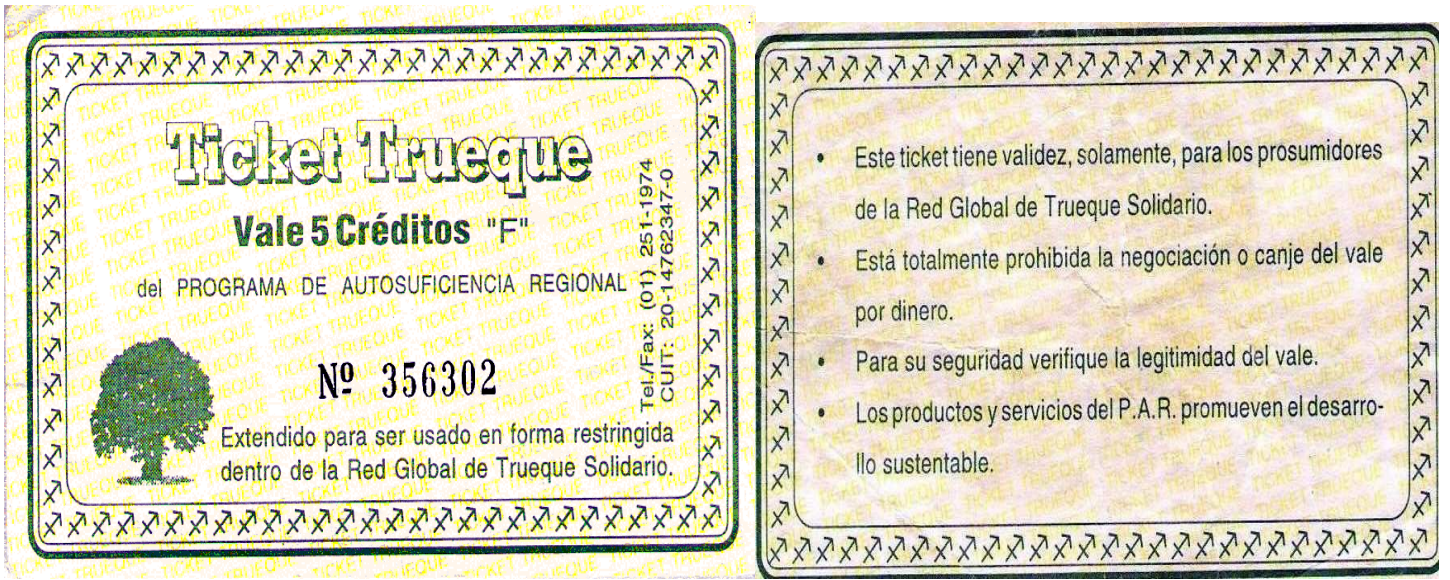

Figuras 1 y 2. Ticket de trueque por valor de 5 créditos equivalente a 5 pesos, correspondiente a la Red Global de Trueque Solidario. Muchas personas conservan gran cantidad de tickets sin ningún valor al desaparecer el fenómeno. Fotografía realizada por la autora del anverso y reverso de un «crédito» de los llamados «del arbolito».

La experiencia comienza a repetirse en la ciudad de Buenos Aires y en la zona norte de la provincia, en Olivos, pero como se hace complicado el manejo del sistema de tarjetas y planillas de cálculo se introduce la edición del «vale de intercambio o crédito». Cuando los intercambios empiezan a darse entre socios de varios clubes y zonas, nace una red de club del trueque: la Red Global de Trueque Solidario, RGT, según el nombre que figura en el formato 
inicial del «ticket trueque». La unidad de cambio es denominada «crédito», con equivalencia a la moneda oficial, 1 crédito=1 peso (Hintze 2003: 52).

Un año después de la inauguración, el primer nodo ya contaba con mil socios. En el año 1996, Hora Clave, programa del célebre periodista Mariano Grondona, dedicó una emisión al fenómeno y a partir de ahí se desató una verdadera eclosión del trueque que alcanzó una dimensión nacional, expandiéndose especialmente en las provincias de Río Negro, Santa Fe, Córdoba y Mendoza. En 1997 son 2.300 socios y en 1999 llegan a 180.000 los participantes. Un año más tarde, el incremento es exponencial: el trueque alcanza a 320.000 personas, reunidas en cerca de 400 nodos ubicados en 15 provincias y la ciudad de Buenos Aires.

En 2001 la cantidad de nodos se duplica y los participantes llegan a medio millón en más de veinte provincias. A comienzos de 2002 se produjo la eclosión, registrándose un impresionante ascenso a partir del impacto producido por la crisis del sistema bancario argentino, que aunó la recesión económica a la disminución notable del dinero circulante, calculándose ya la existencia de cuatro mil quinientos nodos en funcionamiento y de un número de participantes superior a los dos millones. ${ }^{6}$ Antes del establecimiento del «corralito»se registraban en los nodos veinte mil personas por mes, mientras que desde el colapso del sistema financiero se integraron cinco mil socios cada día (Leoni y Luzzi 2003: 17). 


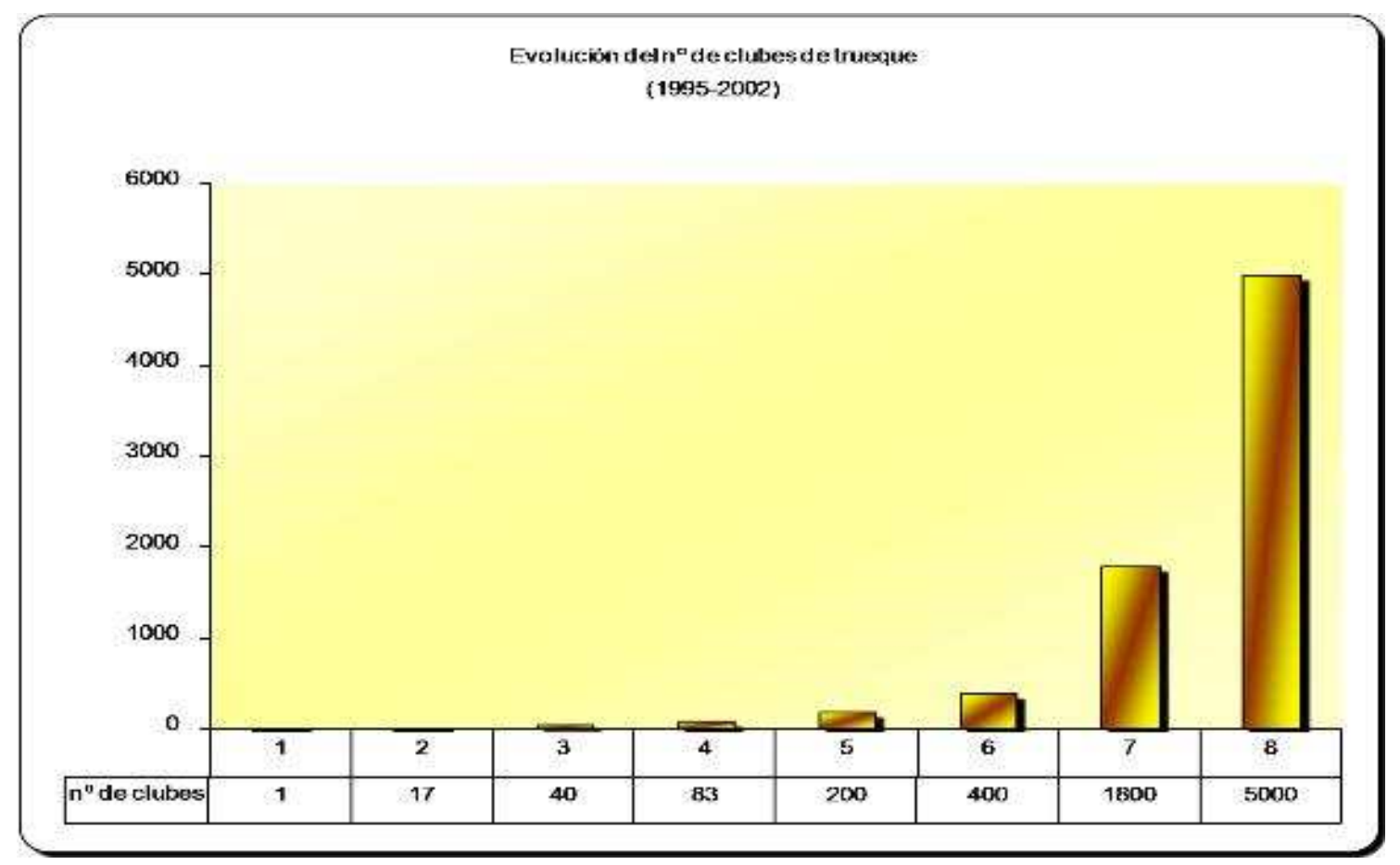

Figura 3. Expansión del número de nodos de trueque en Argentina, desde su origen en 1995 hasta 2002 en que alcanza su máxima expansión en los momentos más agudos de la crisis. Gráfica de realización propia.

Diferencias internas derivaron en dos grandes redes: la Red Global del Trueque, RGT —con sede en Bernal—, y la Red de Trueque Solidario, RTS, que aglutinaba a la mayoría de los nodos del país, cuyo funcionamiento es independiente y horizontal (Stancanelli 2002: 9).

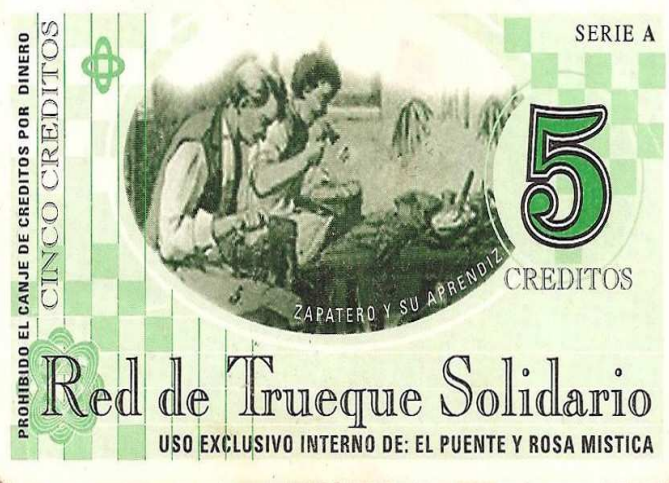

- Este vale no constituye medio de pago.

- Sulo vale como elemento de INTERCAMBIO.

- Prohibido negociar, cobrar comisiones o canjear por dinero.

- Solo es válido para miembros activos de la Red Sulidaria de Trueque.

- No se usa dinero dentro de la Red Global.

- Ilacer válidas estas reglas es tener honestidad hacia la Red Global y hacia nosotros mismos.

Ll día que aprendamos a ceder

empezaremos a ser.

Sello

Figuras 4 y 5 . Anverso y reverso de un ticket por valor de 5 créditos correspondiente a la Red de Trueque Solidario. En esta red se hace especial hincapié en el valor de intercambio y no monetario de los mismos. Fotografías realizadas por la autora. 


\section{VIVIR DEL TRUEQUE}

El club de trueque era una organización de «prosumidores». El concepto de prosumidor se tomó del libro La Tercera Ola, de Alvin Tofller, que define como tal a las personas que son al mismo tiempo productores y consumidores (Hintze 2003: 37). La iniciativa se propagó por todo el país, generando un intercambio incalculable de bienes y servicios que de otro modo hubiera sido difícil de llevarse a cabo.

Los intercambios de bienes y servicios se realizaban en lugares llamados nodos y la moneda que se utilizaba era un papel llamado crédito, que tenía un valor semejante al dinero oficial en Argentina. Este mecanismo de economía alternativa no trató de reemplazar a la economía formal, de la que siempre pretendió formar parte.

Por el contrario, el objetivo era ser un complemento para aquellos que por diversas circunstancias estaban parcial o totalmente fuera del sistema económico, obteniendo a través de esta actividad un insumo, a veces fundamental, para la subsistencia de muchas familias. ${ }^{7}$

Estos clubes se interconectaban configurando una gran red nacional. El éxito creciente del club de trueque en Argentina generó un crecimiento explosivo de la red. La red de trueque se componía de todos los nodos existentes en el país, y en los momentos más agudos de la crisis, durante el año 2002, llegó a aglutinar entre seis y siete millones de personas, para iniciar desde entonces un declive continuado y prácticamente desaparecer; ya que no hemos podido acceder a ninguno desde el año 2003, como era nuestro objetivo, salvo en algunas localidades de ámbito reducido. 


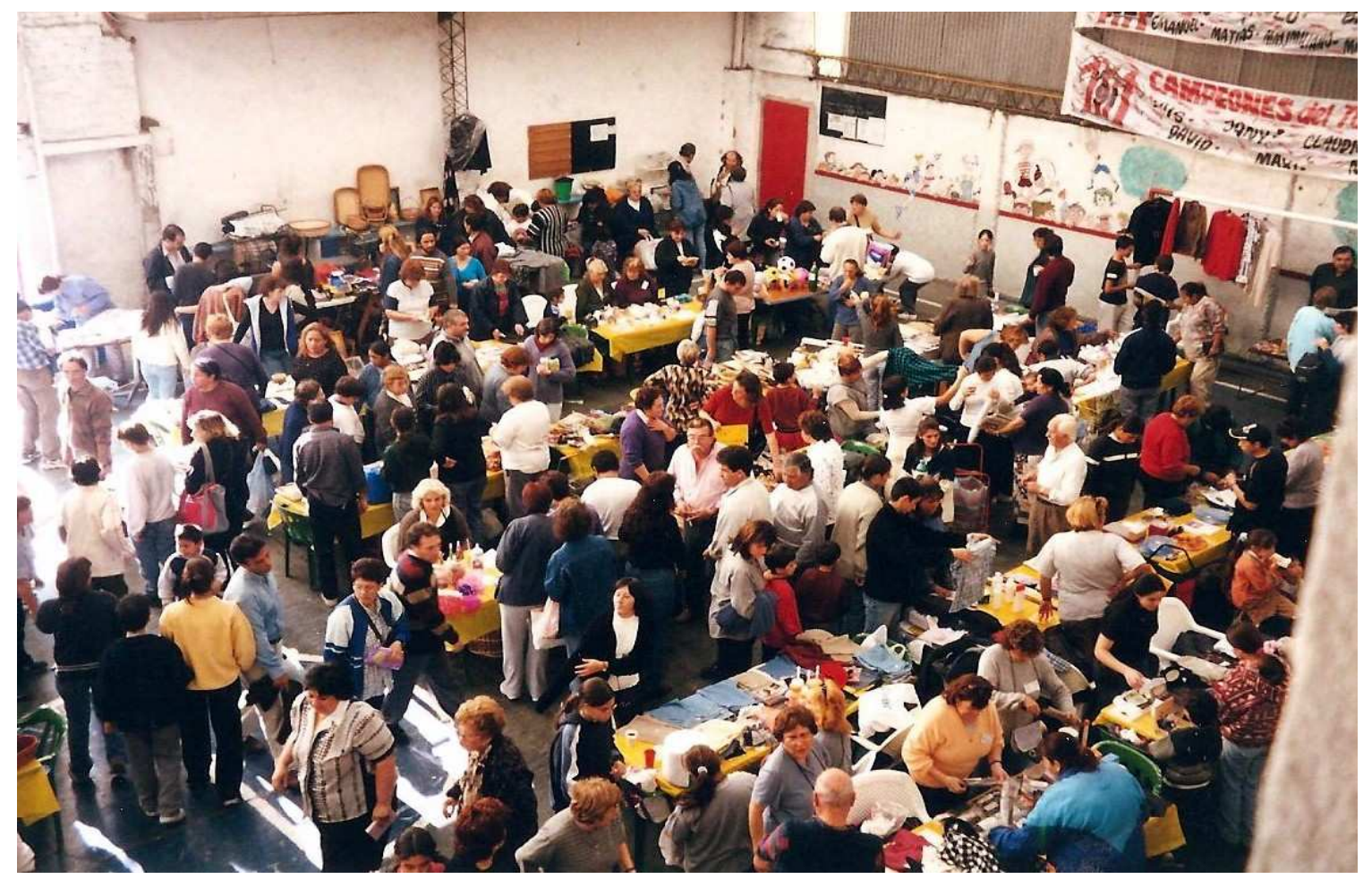

Figura 6. Vista amplia del nodo de trueque situado en calle Primera Junta 876, de Ciudad Madero, en la zona Oeste del conurbano bonaerense, correspondiente al partido de La Matanza. En los momentos de gran apogeo las ferias llegaron a reunir a más de 5000 personas diarias y fue, sin duda, la estrategia que permitió a amplio sectores de la clase media empobrecida poder sortear aquel difícil invierno de 2002.

La importancia que tenía la participación en el trueque y la posibilidad de abastecerse difería según los distintos estratos sociales. En el caso de los sectores medios, que normalmente disponían de ciertos ingresos monetarios y no se encontraban en una situación de extrema necesidad, el uso paralelo del trueque les permitió obtener un mayor rendimiento de sus ingresos, mantener cierto nivel de vida y no caer en la pobreza profunda. Vivían del trueque y podían reservar sus ingresos monetarios para los bienes y servicios que no se conseguían por créditos, por ejemplo para el pago de las cuentas mensuales de gas, luz, etcétera.

Los sectores populares, sin embargo, se insertaron más tarde en el trueque impulsados casi exclusivamente por la urgencia alimentaria. Para ellos el trueque era una estrategia de sobrevivencia sumamente importante, pues se encontraban sin empleo, sin ingresos fijos y les 
resultaba difícil satisfacer las necesidades alimenticias de sus familias. A diferencia de los sectores medios no participaron en el trueque como mercado paralelo, sino casi como la única opción para conseguir algún tipo de ingreso, aunque no fuera en dinero sino en bienes y servicios.
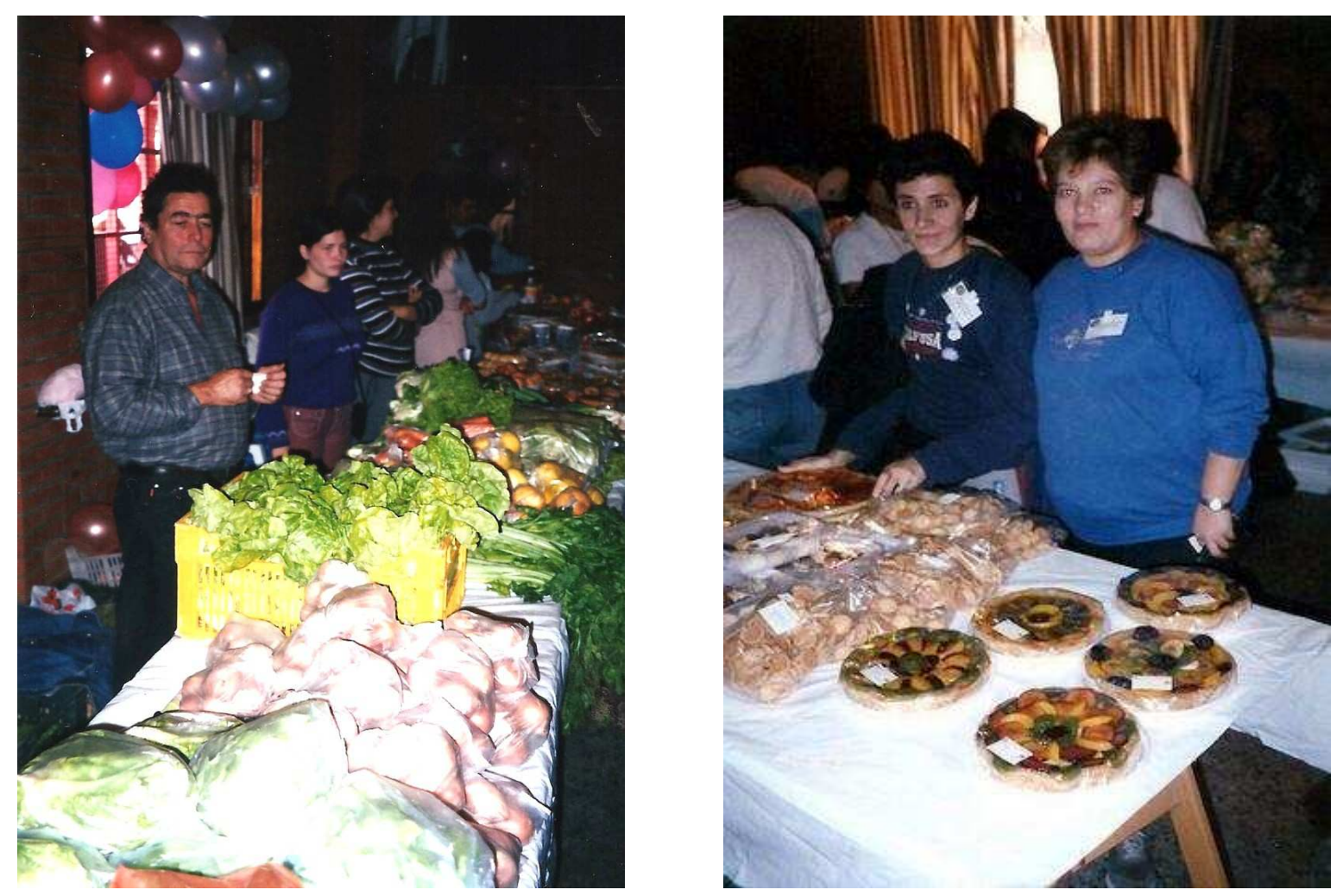

Figuras 7 y 8 . En la imagen de la izquierda diversos prosumidores intercambian frutas y verduras de sus quintas. A la derecha, prosumidoras exponen diversos productos de pastelería y dulces, siendo muy común la oferta de alimentos elaborados — pastas, dulces, zumos, etc.—, mientras que con el tiempo las materias primas para su preparación, como leche, huevos, azúcar, harina, etc., fueron un bien preciado en los nodos difícil de conseguir. Fotos procedentes del nodo de trueque del barrio de Madero, 27 de abril de 2002.

Dentro de este sector del tejido social, el abastecimiento con productos y servicios de la vida cotidiana fue el motivo principal para participar en el trueque. Los prosumidores adquirieron, por ejemplo, alimentos, ropa y servicios: peluquería, tratamiento médico, odontológico, psicológico, trabajos de fontanería, electricidad, etc. De esa manera los hogares podían bajar sus gastos en pesos y en el caso de muchos otros directamente significó poder comer diariamente. 
Así nos cuenta nuestro entrevistado Horacio García, ${ }^{8}$ remisero:

Sí, lo conocí y viví del trueque; en ese momento a mí me salvó, porque yo pude solventar mí casa y al menos darle de comer a mis hijos; porque yo no tenía nada, nada de nada, a mí me sirvió mucho; yo puse lo que yo tenía, lo que sabía ... bueno, yo me dedicaba a llevar artículos eléctricos, que era lo que tenía, y después me dediqué a hacer pasteles de hojaldre que era mi fuerte, entonces iba al nodo y hacíamos el trueque ... fue un momento en que el país estaba muy mal y apareció el trueque, y fue un alivio para todos, y se aliviaba también al gobierno.

Para Elsa Cataldo ${ }^{9}$ y su familia, el trueque fue un mecanismo para subsistir:

A nosotros nos fue bien, vieron ustedes que estuvieron en el Cristo, que estuvieron en la esquina del Cristo [Elsa hace referencia a una imagen tamaño natural de un Sagrado Corazón de Jesús, que preside la entrada al pueblo], bueno, ahí hay una cancha [campo de fútbol], ¿ustedes la vieron? Bueno, ahí íbamos nosotras; nos íbamos de a pie y volvíamos de a pie con la mercadería, en pleno verano a las dos, las tres de la tarde ... Hay que saber moverse, también hay que avivarse y hay que aprender; hay gente que por ejemplo trabaja en SWIFT [fábrica de alimentos], entonces esa gente trae picadillo de carne, trae algún producto cárnico y lo cambia, y hay que saber negociar. Cuando entras, hay que pensar «voy a precisar esto, voy a precisar aquello» ... la que lleva azúcar [que por ahí no es mucha la gente], la que lleva leche, leche en polvo y, bueno, es todo un mundo ahí adentro .... Laura [se refiere a su hija] vendía y yo compraba; Laura vendía, ella iba y vendía las cosas; estaba en un sitio fijo y yo iba recorriendo a medida que ella iba vendiendo, yo me iba proveyendo de lo que necesitaba. Hemos ido a otro trueque en Rosario, pero no era lindo, era más para ropa, para cosas no tan imprescindibles... 


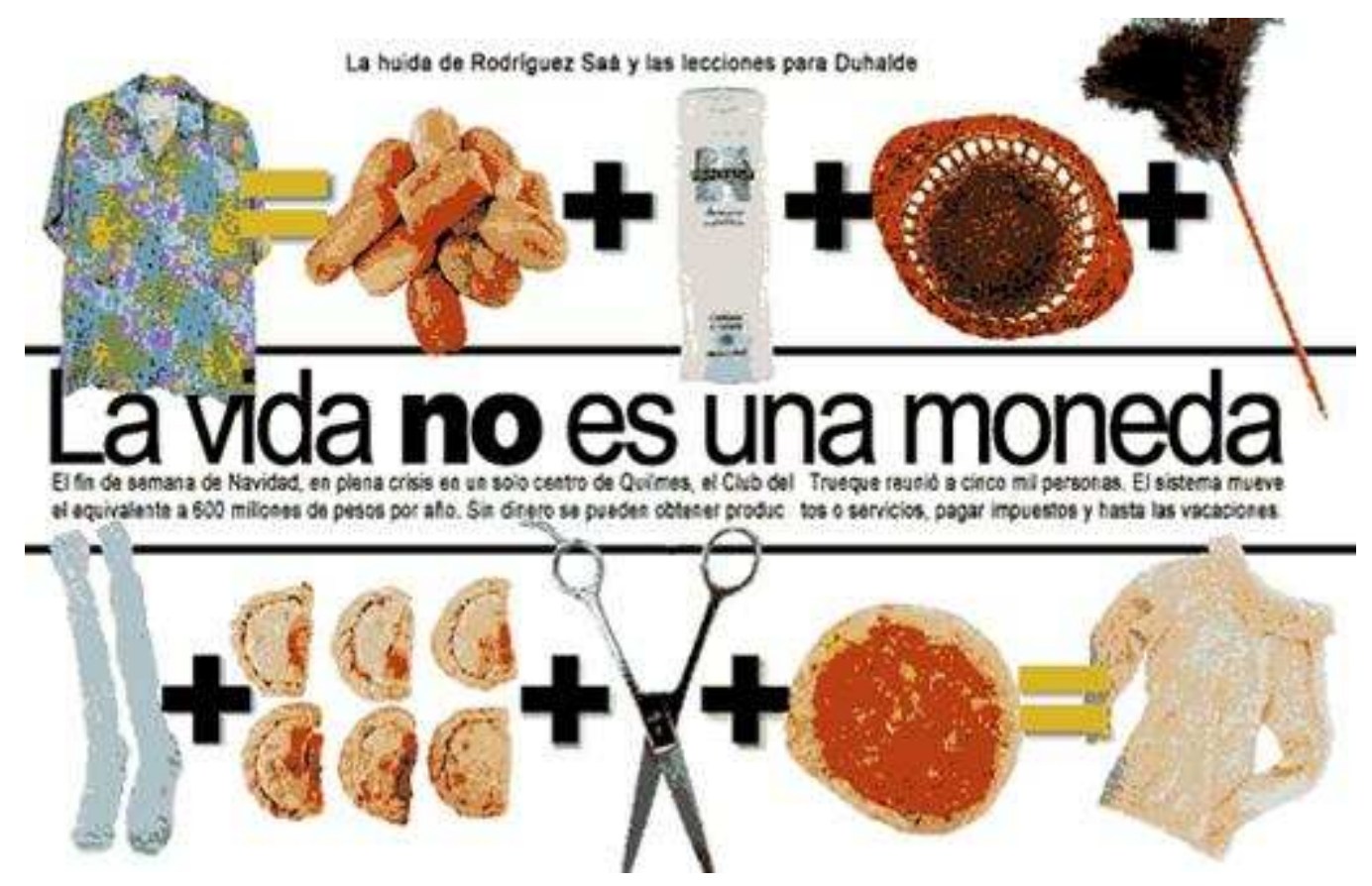

Figura 9. Una imagen de la Revista 23 en donde se habla del trueque; allí se daba cuenta de la importancia del fenómeno y se reseñaba que sin dinero se podían obtener productos o servicios, pagar impuestos y hasta las vacaciones. Portada de Revista 23, Buenos Aires, Argentina. 4 de enero de 2002.

Dora Domínguez, ${ }^{10}$ enfermera y actualmente empleada como personal administrativo, nos describe el proceso seguido en el primer club de trueque creado en San Nicolás, en el barrio

\section{Güemes:}

Yo estuve en el trueque, participé en el trueque también, un año y pico. Yo, por ejemplo, hacía jugos de frutas y cosas de tejidos, porque yo hago artesanías, entonces llevaba eso. Te digo que al principio funcionó muy bien. Cuando éramos poquitos hacíamos trueque directo, después porque empezamos a buscar información vino gente de Buenos Aires y empezaron a circular los ticket, los «tickesitos» que te servían solamente para eso, pero después ya entró la avivada. Nosotros por ejemplo empezamos en el barrio Güemes, en el barrio donde yo trabajaba en el dispensario; empezamos a juntarnos, llamé a una contadora que era amiga, ella viajaba siempre a Buenos Aires; me dice, «mirá, yo tengo conocimiento de lo que hay en Buenos Aires», y empezamos a contactarnos, estuvimos como dos meses reuniéndonos antes de largarlo, fuimos el primer trueque que existió en San Nicolás Te estoy 
hablando de finales de 1999 a 2000, por ahí fue cuando arrancábamos. Fuimos bárbaro, porque era un grupo chico, después se fue agrandando, pero ya con más requisitos, nosotros pedíamos que fueran cosas elaboradas, no una reventa; empezamos con cosas elaboradas tanto en alimentos como en prendas, por ejemplo: licores caseros; todo lo que es pasta, todo casero; lo único que prometíamos que no era elaborado era la verdura, vos te dabas cuenta que era cosechado de su quinta, y así fue cuando se iba agregando más gente, porque empezamos a salir en la televisión, en la radio; lo que empezamos a hacer fue poner ciertos requisitos, pero de golpe fue que apareció, porque a este trueque lo tildaron como que era un trueque del radicalismo. Entonces, qué hicieron, Bancalari, ${ }^{11}$ llamó a un grupo de sus punteros, ${ }^{12}$ de mujeres, y les dijo: «a mí me arman un trueque ya, porque estos laureles se los está llevando el radicalismo». Pero nada que ver, ni siquiera militantes del partido fueron jamás al trueque, y ahí ya se tergiversó, ya se pudrió, porque aparecieron los punteros.

Éramos alrededor de cien personas, era semanal. Y después, claro, salió un trueque acá, otro allá, la cuestión es que había créditos de todos los colores, habidos y por haber, y nosotros llegado un momento llegamos a tener ochocientas personas afiliadas, pero ya no los podías controlar, no te respetaban, no respetaban a los coordinadores, no respetaban los requisitos, iba a terminar siendo una lucha de pobres contra pobres, entonces ... dijimos, no. No va más. Yo estuve un año, el segundo año siguió Ariel, que es el marido de una chica amiga, él tenía un entusiasmo y el siguió otro año más; duro dos años en total.

Hubo corrupción. Uno, por ejemplo, un tipo en la ex fabrica textil La Stella apareció un día hablando que él iba a hacer la feria ahí y que quien quisiera [podía] hacerse socio, claro, en ese galpón una feria terrible y funcionó un mes, se llevó toda la guita y se fue. Hubo muchos casos así ... La gente que venía al nuestro también iba a los otros y a los otros, entonces, traían las mañas de otros sitios que les permitían cosas que nosotros no; por ejemplo, no dejábamos entrar a nadie que trajera cosas compradas, inclusive habíamos arreglado con una óptica, con carnicerías, con veterinarias, con algunos médicos, inclusive con la Clínica Alvear, entonces, ¿qué hacía la clínica? Con los créditos que recibía compraba material para la cocina, repasadores, servilletas, pero principalmente para alimentos pero, claro ... cuando empezaron a agregarse muchos de golpe y traían las mañas de los otros trueques se empezó a venir abajo. Y nosotros no quisimos quedar prendido a todo esto. Una lástima porque era muy bueno, muy bueno; yo me hice mi baño nuevo con el trueque, accedí a un montón de cosas, me 
instalé toda la conexión de gas porque había gasistas profesionales, pero llevaba comida, ropa, para mí fue una muy linda experiencia. Tuvimos tres nodos: Güemes, Pompeya y barrio Alto Verde, o sea, la cabeza y dos subsedes que funcionaban en distintos días.

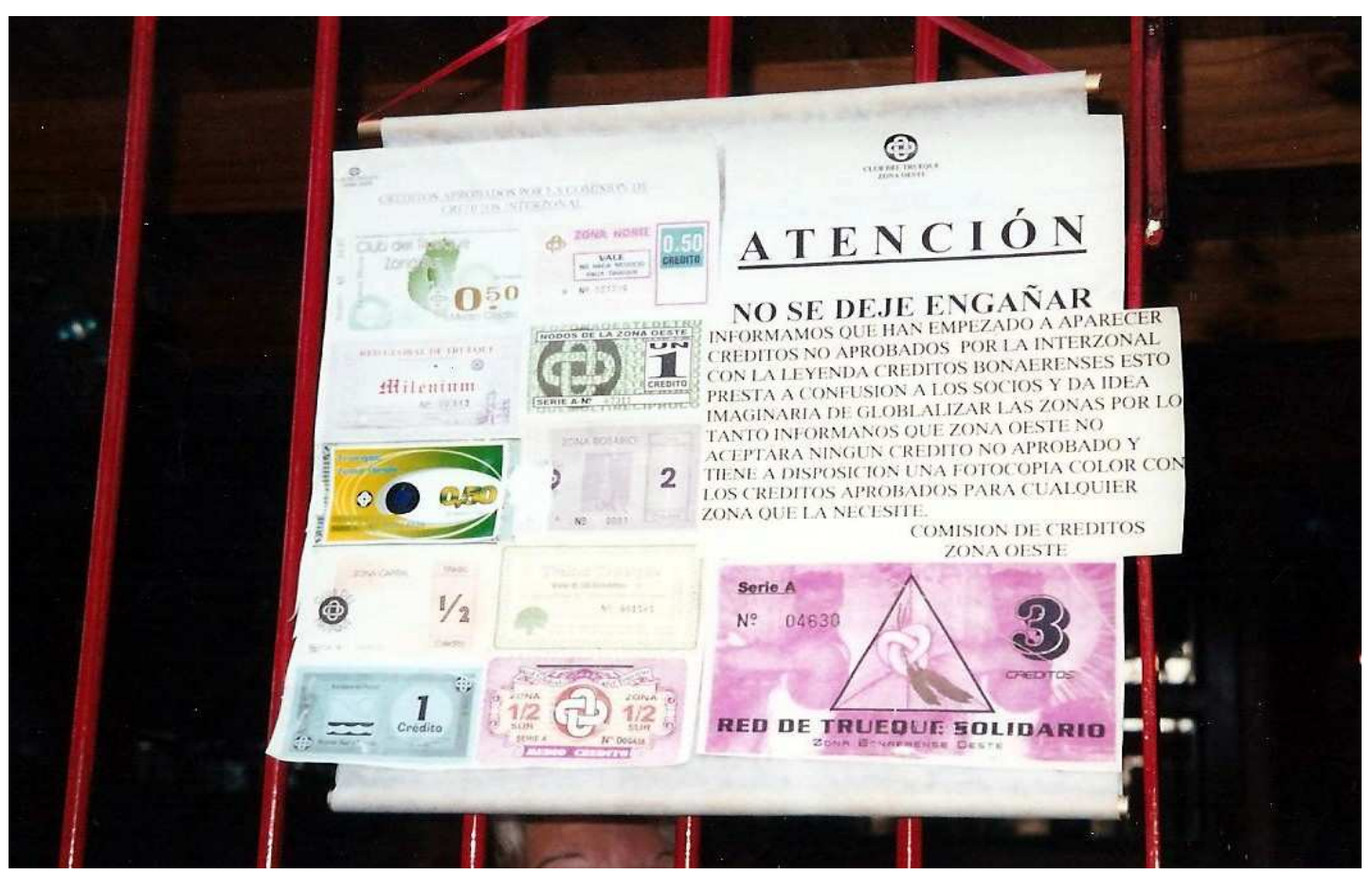

Figura 10. Una de las causas del declive: las falsificaciones de los créditos. En la imagen se muestra la cancela de entrada al nodo de Madero, donde un cartel avisaba en abril de 2002 de la existencia de créditos falsos, informando: «No se dejen engañar, informamos que han empezado a aparecer créditos no aprobados por la interzonal con la leyenda créditos bonaerenses, esto presta a confusión a los socios y da idea imaginaria de globalizar las zonas, por lo tanto informamos que zona Oeste no aceptará ningún crédito no aprobado y tiene a disposición una fotocopia color con los créditos aprobados para cualquier zona que la necesite. Comisión de créditos Zona Oeste». Foto realizada por la autora.

A pesar de la diversidad, el trueque nace principalmente como iniciativa de una clase media empobrecida. No se trata de una pobreza heredada sino adquirida o, mejor dicho, a la que se han visto empujados por el proceso de crisis. Pero, durante el crecimiento del trueque hubo una importante incorporación de sectores con un perfil más próximo a la pobreza estructural. De ahí que se pueda observar la posible relación entre el golpe final que sufrió la actividad del trueque y el lanzamiento y consolidación de los Planes Jefas y Jefes de Hogar Desocupados, observando en qué medida el abandono de los actores del trueque coincidió con la asignación 
de los Planes Jefas y Jefes de Hogar, ya que fueron los colectivos incorporados tardíamente los que más se beneficiaron del Plan. Armando Néstor Tactagi, ${ }^{13}$ dirigente social, comenta al respecto:

Sí, hubo un momento que hubo ... antes de que salieran los Planes; existía ese sector económico informal, no sé cómo llamarlo, que tuvo un desarrollo muy grande; había gente que comía, que c-o-m-ía con el trueque. Bueno, después salieron los Planes, al salir los Planes la gente supo que podía contar con esos $\$ 150$ para poder comer.

A esto se suma la crisis interna que sufrió el trueque relacionada con la desviación de conductas de sus propios actores: los abusos, las falsificaciones en los créditos, la falta de institucionalización; diversos motivos, en definitiva, que llevaron a su declive y que nuestros entrevistados han puesto de manifiesto. Así Ana María Oliva ${ }^{14}$ nos comparte sus impresiones sobre el trueque y las razones de su prácticamente desaparición:

En un primer momento sí, porque la gente lo tomó en serio. Pero sabés que esos bonos con que se acreditaba, de pronto qué sé yo, un peluquero decía, «yo tres veces por semana voy a cortar el pelo gratis», se le daban unos créditos de papel como si fuera dinero y con eso por ejemplo podía comprar comida, o artículos de limpieza, ropa o lo que fuera. El que tenía algo para dar, bien, y el que no brindaba un servicio.

Bueno, empezaron a falsificar esos papeles, entonces tenían papeles falsificados, ya que no eran papeles pasados por la Casa de la Moneda, con el sello y hologramas y todas las medidas de seguridad, y entonces también fracasó eso y empezaron a meter cosas que no estaban permitidas y, bueno, queda muy poco. En general, los que quedan por ahí son de comunidades muy pequeñas, el trueque entre conocidos. En lugares muy grandes, donde iba todo el mundo, alguien llevaba por ejemplo una torta, le daban un papelito que era un crédito, y con ese crédito a lo mejor compraba un champú. Eso funcionaba bastante bien, sobre todo funcionó entre gente de clase media, que siempre tenía algo 
para llevar o para dar o alguna profesión para ofrecer. No fue tan importante con la gente más pobre o más carenciada porque en realidad lo que tenían para ofrecer era nada, casi nulos en servicios y objetos ninguno.

\section{Cristina Irrazábal ${ }^{15}$ nos ofrece también su testimonio:}

Yo lo tenía conocido de Buenos Aires, cuando empezaba a hablarse de los microemprendimientos, empezaba a decirse que había un club de trueque, porque todo estaba muy mal; entonces el odontólogo no conseguía pacientes, el contador le cerraban los negocios, por lo tanto, perdía clientes. Entonces se empezó esto del trueque; yo te atiendo, vos dame los productos de tu huerta; después vino alguien que lo sistematizó, creó como una red, creó unos vales que tenían un valor simbólico para facilitar el trueque, ya sabemos cuál es la palabra trueque, te cambio mis anteojos por tu reloj.

Empezó en algún galpón de Buenos Aires, yo nunca lo tuve muy en cuenta. Una vez acá en San Nicolás empezó en la iglesia de Pompeya, y yo tenia unos cuantos juguetes que me habían quedado de un negocio que había tenido y me dije «bueno, vienen las fiestas, los Reyes y Papa Noel», y se me ocurrió llevarlos a ese trueque.

Tuvimos unas charlas introductorias, te enseñaban cómo calcular, un poco a ponerle precio. Hacían hincapié y pretendían que esto fuese algo de producción, que la persona lo tuviera como un trabajo. Que la persona lo elabore o produzca, que se cree como un trabajo y vaya e intercambie con otros y evitar el tema de la comercialización, sin mayoristas.

El que tenga dinero, de pronto va y compra un montón de zapatillas y las vende como una boca de expendio. En la realidad, eso igual sucedía porque todos necesitan calzado o alguien le gusta tomar sus vinos y entonces hay todo de todo.

\section{Margarita Pardo, ${ }^{16}$ ama de casa, comenta:}

Sí, yo estuve mucho tiempo con el trueque, muy bueno, muy bueno. Cuando yo estaba, llevaban milanesas de soja, a veces compraban las cosas; compraban papel higiénico y otras cosas, yo lo dirigía con mi yerno ... en Luz Verde, pero después se pudrió todo por los ladrones ... llegó un momento que 
era incontrolable, no había manera de controlar a la gente, entraban setecientas personas ahí en el salón de la escuela ... pero era una cosa que era imposible de mantener...

Yo me acuerdo que nosotros fuimos a la fiesta que se hizo en Buenos Aires, nos pusieron un colectivo, fuimos a una sola que no me acuerdo el nombre, porque yo tengo mala memoria ... Una fiesta hermosa con las tres personas que iniciaron el trueque, en ese lugar estuvieron artistas, inauguraron unos bustos de esas tres personas juntas ... estos tres que decían que inauguraron el trueque, pero ¿sabe? El principal que se fue a España, porque se fue a España, porque era así: hacerte socio costaba $\$ 3$ y te daban una determinada cantidad de tickets, todo ese dinero nosotros lo teníamos que entregar porque no se usaba dinero, nosotros teníamos créditos ... bueno, todo se mandaba allá. Aquí traían de todo ... pero en otros lugares llevaban muchos animales ... gatos ... ah, en Mendoza ... se podía vivir, podías vivir ... pero cuando empezó, todo esto se pudrió ... Era una avalancha, iba a trocar o a vender o a comprar ... pero ¿qué pasaba? ... se armaban problemas, porque vos estabas en cola horas y horas ... cien personas, doscientas personas para entrar, entonces desde la cola unos a otros se encargaban cosas, de modo que algunos al llegar ya no encontraban nada. Y uno les decía «no encarguen, no encarguen, las cosas se compran en los mostradores» ... pero no había caso ... no había forma ... llega un momento que la gente es incontrolable.

\section{O como expresa Valeria Oberti: ${ }^{17}$}

Al principio sí, funcionaban bien; compraban bien, pero después ya la gente robaba; por ejemplo había un criadero de gallinas o huerta y la gente robaba y la llevaba al trueque, y así fue desapareciendo todo; la gente tenía que invertir igual, pero bueno, fue una alternativa a la crisis; en otros lugares ha funcionado bien, podían acceder a carne, a cosas de valor ... ¡Una persona hasta se compró un auto con el trueque!, no acá en San Nicolás, por la zona de Junín, Salto, en esa zona ... porque había una red de trueque entre las localidades.

En un contexto en que la cantidad de socios se triplica debido a la crisis del país, la cantidad de moneda social circulante se desborda y crece el problema de lo que para algunos será la 
falsificación y para otros la sobreemisión de créditos. A mediados de 2002 se afirma que 30\% de los créditos en circulación eran falsos.

Dos de los creadores del sistema, Rubén Ravera y Horacio Covas — coordinadores de La Bernalesa-, afirmaron que el presunto fracaso respondió a una serie de factores:

... una enorme falsificación de créditos, la inflación en los precios, un ataque de ciertos medios de prensa y la especulación de aquellos que conseguían alimentos u otros elementos indispensables para una familia. Igual, no hablan de fracaso, sino de crisis de crecimiento» $^{18}$.

Un informante anónimo ${ }^{19}$ nos decía:

Lo desvirtuaron del todo, completamente, y .. ¡ isomos argentinos!

Empezaron a hacer unos tickets que eran falsos, empezó a ir gente que tenía negocios como yo, iban y me lo decían a mí: yo compro papel higiénico a 20 y voy allí y lo vendo a 35, y hago el trueque; y entonces lo desvirtuaron; no era eso, esto tenía que haber sido una fórmula cooperativa; bueno, tenía que haberlo implementado el sistema político.

El padre Marcelo Doménech, ${ }^{20}$ párroco de la iglesia de Nuestra Señora de Pompeya, donde funcionó uno de los trueques más activos de San Nicolás, nos comenta sobre el final de la experiencia:

Fue interesante, pero estamos tan metidos en la corrupción que la gente misma, algunos, yo te diría delincuentes, utilizaron el trueque para poder lograr sus propias ganancias. Una cosa increíble, porque lo del trueque tenía todo un pensamiento buenísimo: el compartir, el respeto, el confiar. El trueque lo que trajo, además de poder solucionar algunos problemas de tipo económico inmediato, traía la confianza; yo confió en vos, porque tu producto es bueno, y vos confiás en mí, porque mi producto es bueno. Y entonces intercambiábamos el producto de lo que yo podía hacer, les hacia sentirse útiles y, además, si no nos conocíamos empezábamos a conocernos y a lograr una buenísima relación. 
Pero apareció gente que utilizó el trueque para su propia ganancia y hacía negocios allá, compraban más barato allá, vendían más caro acá y recorrían, hubo gente que con la ganancia del trueque se viajó a Europa. Una locura.

El fenómeno del trueque ya no reviste la importancia de años atrás, pero constituyó la estrategia de contención más generalizada y efectiva que junto a los Planes Jefas y Jefes de Hogar Desocupados proporcionaron soluciones alternativas capaces de evitar el desborde social, y actualmente representa un referente para otros países latinoamericanos insertos en

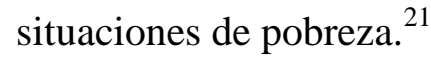




\section{BIBLIOGRAFÍA}

Almeyra, Guillermo, 2004, La protesta social en la Argentina (1990-2004). Ediciones Continente, Buenos Aires.

Altimir, Óscar y Luis Beccaria, 1998, Efectos de los cambios macroeconómicos y de las reformas sobre la pobreza urbana en la Argentina. Documentos de Trabajo de la Universidad General Sarmiento, mimeo. Buenos Aires.

«Boletín No Nos Llamamos Plata, núm. 41», 2006, Revista digital Autosuficiencia, diciembre. Buenos Aires. http://tabloide.eurofull.com/shop/detallenot.asp? notid=971 [consulta: junio, 2008].

Coraggio, José Luis, 1998, «Las redes del trueque como institución de la economía popular». En Economía Popular Urbana: Una nueva perspectiva para el desarrollo local. Cartillas del Programa de Desarrollo Local, núm. 1, octubre. UNGS, Buenos Aires.

De Sanzo, Carlos, 1998, «Todo empezó con un zapallo a media noche: la historia oficial». En Reinventando el mercado: la experiencia de la Red Global de Trueque en Argentina, Heloísa Primavera, Horacio Covas y Carlos de Sanzo (eds.). Ediciones PAR, Buenos Aires.

De Sanzo, Carlos y Horacio Covas, 1997, Clubes de Trueque. Una alternativa de fin de siglo. Programa de Autosuficiencia Regional, Bernal, Buenos Aires.

De Sanzo, Carlos, Horacio Covas y Heloisa Primavera, 1998, Reinventando el mercado: la experiencia de la Red Global de Trueque en Argentina. Programa de Autosuficiencia Regional, Bernal, Buenos Aires.

Ezcurra, Ana María, 1997, «¿Qué es el neoliberalismo? Evolución y límites de un modelo excluyente». En Ideas. Instituto de Estudio y Acción Social, Lugar Editorial, México. 
Fernández Mayo, Manuela, 2006, Historia actual de la Argentina a través de la oralidad. Crisis y estrategias. 2 tomos. Universidad de Cádiz, AAT 3198021, Cádiz.

Hintze, Susana (coord.), 2003, Trueque y economía solidaria. Buenos Aires, Prometeo Libros, Buenos Aires.

Iglesias, Mariana, 2002, «Con el trueque ya se compran campos, autos y hasta casas». Clarín, sección Sociedad, p. 18, 14 de febrero. Buenos Aires, Argentina.

Instituto Nacional de Estadísticas y Censos, INDEC, 2003, «Incidencia de la pobreza y la indigencia en los aglomerados urbanos. Octubre 2002». En INDEC, Buenos Aires, 31 de enero. http://www.indec.gov.ar/nuevaweb/cuadros/74/ pob_toct02.pdf [consulta: marzo, 2008].

Leoni, Fabiana y Mariana Luzzi, 2003, «Nuevas redes sociales: los club de trueque». En Respuestas de la sociedad civil a la emergencia social. Ed. CEDES, Centro de Estudios de Estado y Sociedad, Buenos Aires.

Minujín, Alberto (coord.), 1993, Cuesta Abajo. Los nuevos pobres: efectos de la crisis en la sociedad argentina. UNICEF, Losada, Buenos Aires.

Minujín, Alberto y Gabriel Kessler, 1995, La nueva pobreza en Argentina. Planeta, Buenos Aires.

Ovalles, Eduardo, 2002, Argentina es el país del mundo en el cual el fenómeno del trueque tiene mayor dimensión social. Centro de Estudios Nueva Mayoría, Buenos Aires.

Primavera, Heloisa, 1998, «Unicornios: entre la Utopía y la responsabilidad social. La experiencia del trueque en Argentina». En Expanding people's spaces in globalising economy. Hanasaari, Finlandia. 
2001, Cómo comenzar una red de trueque solidario. RedLASES, Buenos Aires.

Rosanvallon, P. y J.-P. Fitoussi, 1997, La nueva era de las desigualdades. Manantial, Buenos Aires.

Rossmeissl, Bárbara, 2005, «El Trueque en Argentina-¿Estrategia eficiente en tiempos de crisis?». Revista de Economía, Buenos Aires. http://www.eumed.net/cursecon/ecolat/ar/2005/br-trueque.htm [consulta: octubre, 2007].

Sainz, Alfredo, 2002, «El aumento de los precios, la caída del empleo y los ingresos llevaron al $80 \%$ de las familias a comprar sólo lo indispensable». La Nación, sección Economía, p. 1, 1 de diciembre. Buenos Aires, Argentina.

Seoane, María, 2003, El saqueo de la Argentina. Editorial Sudamericana, Buenos Aires.

Stancanelli, Pablo, 2002, «Cuando el Estado ya casi no existe. Explosivo crecimiento de los clubes de trueque». Le Monde diplomatique, núm. 36, junio. Edición Cono Sur, Buenos Aires, Argentina.

Svampa, Maristella (comp.), 2003, Desde abajo. Las transformaciones de las identidades sociales. Biblos, Buenos Aires.

Wacquant, Loïc, 2001, Parias urbanos. Marginalidad en la ciudad a comienzos del milenio. Ed. Manantial, Buenos Aires.

Zibechi, Raúl, 2003, Genealogía de la revuelta Argentina: la sociedad en movimiento. Nordan Comunidad, Montevideo. 


\section{Notas}

${ }^{1}$ Según datos del Instituto Nacional de Estadísticas y Censos, INDEC, en Argentina 20,8 millones de personas vivían en la pobreza y 9,96 millones de éstos en la indigencia. INDEC, «Incidencia de la pobreza y la indigencia en los aglomerados urbanos. Octubre 2002». En INDEC, Buenos Aires, 31 de enero de 2003. http://www.indec.gov.ar/nuevaweb/cuadros/74/pob_toct02.pdf [consulta: marzo, 2007].

${ }^{2}$ Durante los últimos meses previos a la renuncia del ex presidente De la Rúa, los indicadores sociales estaban muy deteriorados. La suspensión de pagos a la deuda externa y la devaluación de la moneda hicieron que la economía fuera rápidamente desestabilizada y que la inflación sufriera un fuerte ascenso. Las primeras consecuencias fueron la disminución e incluso la pérdida de los ingresos familiares y, por ende, un aumento vertical de la pobreza y de la indigencia que alcanzaron índices sin precedentes hasta $57.4 \%$ y $27.5 \%$ de la población, respectivamente, en octubre de 2002. Fernández Mayo, Manuela, 2006, Historia actual de la Argentina a través de la oralidad. Crisis y estrategias. Universidad de Cádiz, España, AAT 3198021. p. 287.

${ }^{3}$ El 30 de noviembre de 2001 se decretó el congelamiento de los depósitos bancarios dejando atrapados los ahorros de buena parte de la clase media dentro del ya tristemente célebre «corralito» — por alusión al pequeño recinto donde pueden jugar los niños que todavía no andan-, nombre popular que recibió la medida de restricciones bancarias impuestas por decreto por el gobierno del presidente Fernando de la Rúa — liderado por Domingo Cavallo- con el propósito de retener en los bancos los pesos y los dólares, evitando la «corrida bancaria». La norma establecía que los argentinos solo podían retirar 250 pesos o dólares por semana, 1,000 por mes, lo que provocó un tremendo malestar en la clase media, pero también en aquellos que vivían de la economía informal. El gobierno sostuvo que de esta forma, además de evitarse la caída de los bancos, se estimularía la «bancarización» de la economía, ya que los particulares y las empresas se verían obligados a pagar con tarjetas de crédito o débito y con cheques. Afirmaba también que la bancarización contribuiría a reducir la evasión impositiva. Sucedió todo lo contrario. Ibídem, Fernández Mayo, Manuela, 2006, Historia actual de la Argentina a través de la oralidad, p. 144.

${ }^{4}$ Durante la crisis económica mundial de los años 30, la municipalidad del pequeño lugar austriaco de Woergl introdujo una moneda local con la cual la alta tasa de desempleo se pudo reducir $25 \%$ en menos de un año. En Alemania, tras la Segunda Guerra Mundial se fundaron varios clubes de trueque, sobre todo en el sur del país. Ellos practicaron el trueque directo o también a través del ticket, y contribuyó a mejorar la situación de la población participante. Sin embargo, el trueque argentino tiene una mayor dimensión. Rossmeissl, Bárbara, 2005, «El Trueque en Argentina-¿Estrategia eficiente en tiempos de crisis?». Revista de Economía, Buenos Aires, Argentina. http://www.eumed.net/cursecon/ecolat/ar/2005/br-trueque.htm [consulta: febrero, 2007].

${ }^{5}$ Hubo un hecho que lo hizo reflexionar. Durante un tiempo le regaló a una vecina los zapallos que le sobraban, ella se dedicó a hacer dulces y se convirtió en una pequeña empresaria. Carlos de Sanzo se planteó qué pasaría si se reunieran muchas personas e hicieran lo mismo que su vecina, es decir, aprovechar lo que cada uno tiene y a lo que no puede sacarle provecho por falta de trueque. Esa fue la idea para el primer club de trueque argentino, que comenzó su andadura el $1 .^{\circ}$ de mayo de 1995. Hintze, Susana (coord.), 2003, Trueque y economía solidaria, p. 166. Ed. Prometeo, Buenos Aires, Argentina.

6 Iglesias, Mariana, 2002, «Con el trueque ya se compran campos, autos y hasta casas». Clarín, sección Sociedad, p. 18, 14 de febrero. Buenos Aires, Argentina.

${ }^{7}$ Sainz, Alfredo, 2002, «El aumento de los precios, la caída del empleo y los ingresos llevaron al $80 \%$ de las familias a comprar sólo lo indispensable». La Nación, sección Economía, p. 1, 1 de diciembre. Buenos Aires, Argentina.

${ }^{8}$ Entrevista a Horacio García, 30 años, oriundo de Nogoyá, Entre Ríos, casado, 4 hijos, remisero. Los remiseros son similares a los taxistas, en su mayoría desempleados de las empresas privatizadas durante la década menemista que invirtieron sus indemnizaciones en comprar un coche para servicio público. A diferencia del taxi, salen de unos locales o remiserías y al llegar a destino por radio la telefonista indica el coste del trayecto. Entrevista, 26 de julio de 2004 en San Nicolás, Bs. As., tras utilizar sus servicios desde el barrio 7 de septiembre o FONAVI al centro de la ciudad.

${ }^{9}$ Entrevista a Elsa Cataldo de Chávez, 46 años, nació en Rosario, Santa Fe; trabaja como cocinera en el comedor escolar de su barrio, Ghiglione, en Villa Gobernador Gálvez, al sur de la provincia de Santa Fe. Su marido escayolista y sus tres hijos se encontraban desempleados, aun así son profundamente solidarios y crían desde los dos años a Sol Jimena, una niña de ahora seis años, hija de unos vecinos del barrio, discapacitada psíquica y física. Entrevista realizada en su casa el día 11 de agosto de 2004.

${ }^{10}$ Dora Domínguez nació en Avellaneda, Buenos Aires, el 28 de noviembre de 1964. Trabajó como enfermera en la Clínica Alvear de San Nicolás, Bs. As., hasta su cierre en 2002; ejerció actividades de trueque y trabaja 
como administrativa del Centro de Estudios e Investigación de la Mujer, CEIM, de San Nicolás, situado en la calle Garibaldi 375. Entrevista realizada el día 6 de agosto de 2004.

${ }^{11}$ José María Díaz Bancalari, abogado de San Nicolás que ha desempeñado durante su trayectoria diversos cargos políticos e institucionales dentro del partido justicialista; fue intendente de San Nicolás, senador, diputado de la Provincia de Buenos Aires y presidente del Bloque Justicialista Bonaerense en el Congreso hasta 2005. Alineado en la corriente duhaldista, fue perdiendo peso político con Kirchner en las elecciones legislativas de octubre de 2005, para alinearse posteriormente con el kirchnerismo, siendo desde 2007 vicepresidente primero del bloque de Diputados Nacionales del Frente para la Victoria.

${ }^{12}$ Punteros políticos, argentinismo que designa a la persona de confianza del político en el barrio, siendo la persona que ofrece intercambio de «favores por votos», constituyen la base del clientelismo político.

13 Armando Néstor Tactagi, 57 años, nació el 5 de junio de 1947 en La Plata, Bs. As.; residente desde hace 15 años en San Nicolás de los Arroyos, es desocupado y coordinador de la agrupación piquetero Corriente Clasista y Combativa, CCC, de San Nicolás, uno de los principales movimientos dentro de los desocupados. Entrevista realizada en la sede de la Asociación de Trabajadores del Estado, ATE, situada en calle Mitre 620 de San Nicolás, 30 de agosto de 2004.

${ }^{14}$ Ana M. ${ }^{a}$ Oliva Martínez, de 55 años, natural de Buenos Aires, capital federal, docente desde hace más de treinta años, ejerciendo en escuelas parroquiales; en Primaria, como maestra; en Secundaria y Bachillerato como profesora de Filosofía, Lógica y Psicología; ahora se dedica exclusivamente a su cargo de Secretaria de Escuela Domiciliaria. Entrevista realizada en Buenos Aires el 17 de agosto de 2003.

${ }^{15}$ Cristina Irrázabal, 54 años, natural de San Nicolás, profesora de inglés y psicóloga social, desempleada de larga duración. Trabajó como traductora en importantes empresas internacionales y en la Comisión de Energía Atómica en Buenos Aires, intentó diversos microemprendimientos que fracasaron. Entrevista realizada en San Nicolás, Buenos Aires, el 20 de agosto de 2003.

${ }^{16}$ Margarita Rosa Pardo nació en San Juan el 9 de julio de 1947, viuda y madre de siete hijos; vive con su compañero desde hace treinta y dos años, ex empleado de Somisa, de origen suizo, que según sus propias palabras malgastó la indemnización. Entrevista realizada el 26 de julio de 2004, en el dispensario médico del barrio 7 de Septiembre de San Nicolás, Buenos Aires, más conocido como barrio Instituto de la vivienda de Buenos Aires, IVBA.

${ }^{17}$ Valeria Oberti, 29 años, nació en San Nicolás, Buenos Aires. Trabajadora Social de la Escuela Privada de Educación Especial «Camino» de San Nicolás — prestigioso centro que también trabaja con alumnos becados por el Estado-. Entrevista realizada en San Nicolás de los Arroyos, Buenos Aires, el 19 de agosto de 2004.

${ }_{18}$ Declaraciones realizadas en un comunicado dado a conocer en una de las páginas oficiales del trueque en Internet: http://www.truequeenlinea.com.ar [consulta: noviembre, 2007].

${ }^{19}$ Entrevista núm. 28 correspondiente a un informante anónimo, que nació en San Nicolás, Bs. As., el 2 de diciembre de 1939, donde regenta un «kiosco», esto es, un pequeño negocio de golosinas, material escolar y objetos de consumo frecuente. Entrevista realizada el 30 de diciembre de 2003 en su negocio.

${ }^{20}$ Entrevista a Marcelo Doménech, natural de Rosario. Nació el 25 de septiembre de 1946, sacerdote, vive en la Parroquia de Nuestra Señora del Rosario de Pompeya, situada en la calle Nación 563, de San Nicolás, Bs. As., siendo su párroco. Persona con una gran trayectoria de compromiso social y desde cuya parroquia se encauzó la organización de uno de los principales trueques que funcionaron en la ciudad. Entrevista realizada el 27 de diciembre de 2003 en el Convento de las Carmelitas de San Nicolás de los Arroyos, Buenos Aires.

${ }^{21}$ El Gobierno Bolivariano, tomando experiencias de Argentina y Colombia, está impulsando en las diferentes comunidades del país el Sistema de Intercambio Solidario, que busca destinar un pequeño porcentaje de la producción al mercado organizado por la localidad, originando así una economía socialmente justa y no condicionada por el dinero, aunque no niegue la existencia de la moneda y sí potencia los valores locales. En Venezuela, el Ministerio para la Economía Popular, MINEP, a través del Instituto Nacional de Desarrollo de la Pequeña y Mediana Industria, Inapymi, con la colaboración de representantes de la Red Global del Trueque, Argentina-Colombia, dieron inicio al Plan Piloto del Sistema de Intercambio Solidario, en los estados Carabobo, Nueva Esparta y Aragua y en el Distrito Capital. «Boletín No Nos Llamamos Plata, núm. 41», diciembre 2006. En Revista digital Autosuficiencia. http://tabloide.eurofull.com/shop/detallenot.asp?notid=971 [consulta: marzo, 2007].

Fecha de recepción: 01 de abril de 2008.

Fecha de aceptación: 31 de octubre de 2008. 